\title{
Exogenous Pulmonary Surfactant as a Vehicle for Antimicrobials: Assessment of Surfactant-Antibacterial Interactions In Vitro
}

\author{
Alexei Birkun \\ Department of Emergency Medicine and Anesthesiology, Crimea State Medical University, Lenin Avenue 5/7, \\ Simferopol 95006, Ukraine \\ Correspondence should be addressed to Alexei Birkun; birkunalexei@gmail.com
}

Received 31 January 2014; Revised 3 April 2014; Accepted 9 April 2014; Published 29 April 2014

Academic Editor: Yi Zuo

Copyright (C) 2014 Alexei Birkun. This is an open access article distributed under the Creative Commons Attribution License, which permits unrestricted use, distribution, and reproduction in any medium, provided the original work is properly cited.

\begin{abstract}
Owing to its unique surface-active properties, an exogenous pulmonary surfactant may become a promising drug delivery agent, in particular, acting as a vehicle for antibiotics in topical treatment of pneumonia. The purpose of this study was to assess a mutual influence of natural surfactant preparation and three antibiotics (amikacin, cefepime, and colistimethate sodium) in vitro and to identify appropriate combination(s) for subsequent in vivo investigations of experimental surfactant/antibiotic mixtures. Influence of antibiotics on surface-active properties of exogenous surfactant was assessed using the modified Pattle method. Effects of exogenous surfactant on antibacterial activity of antimicrobials against Staphylococcus aureus, Klebsiella pneumoniae, and Pseudomonas aeruginosa were evaluated using conventional microbiologic procedures. Addition of amikacin or cefepime to surfactant had no significant influence on surface-active properties of the latter. Obvious reduction of surface-active properties was confirmed for surfactant/colistimethate composition. When suspended with antibiotics, surfactant either had no impact on their antimicrobial activity (amikacin) or exerted mild to moderate influence (reduction of cefepime bactericidal activity and increase of colistimethate bacteriostatic activity against $S$. aureus and P. aeruginosa). Considering favorable compatibility profile, the surfactant/amikacin combination is advisable for subsequent investigation of joint surfactant/antibacterial therapy in animals with bacterial pneumonia.
\end{abstract}

\section{Introduction}

The success of pneumonia treatment in large part is defined by effectiveness of antimicrobial therapy. In turn, efficiency of antibacterial therapy depends on formation of sufficient antimicrobial drug concentration in the site of infection [1]. Direct administration of antibiotics into a tracheobronchial tree using nebulization or intratracheal instillations can provide high concentration of drug in a focus of infection with low level of systemic absorption and, therefore, decreased systemic toxicity [2]. However, in patients with pulmonary diseases directly administered drug may predominantly accumulate in central rather than in peripheral airways. This negative effect is attributable to physiologic properties of fluid distribution in respiratory pathways and to specific inflammatory alterations such as bronchial hypersecretion, bronchoconstriction, and bronchial edema [3-5].
It was hypothesized that use of exogenous pulmonary surfactant as a vehicle for antibacterial agents could enhance the efficiency of local antimicrobial therapy in pneumonia $[6$, 7]. Pulmonary surfactant is a lipoprotein complex produced by cells of respiratory tissue that ensures a variety of essential physiologic functions [8]. One of the most important features of surfactant is the ability to decrease a surface tension, thus providing normal alveolar ventilation and gas exchange and preventing alveolar collapse [9]. It has been shown that, owing to its specific composition, an exogenous surfactant promotes effective and uniform peripheral distribution of fluid in lungs [6]. Furthermore, given the fact that exogenous pulmonary surfactant is able to reexpand atelectatic areas, it is anticipated that, after local administration with surfactant, antimicrobials will deposit in effective concentrations even in collapsed areas of lungs which are more likely to be infected [5]. 
Despite the theoretical feasibility for use of exogenous surfactant as a vehicle for antimicrobials, this method is still not realized in clinical settings. Scientific achievements in this area are limited to several preclinical studies $[6,7$, 10-13]. These studies have shown that interactions between exogenous surfactant and antibiotics are possible. Therefore, alterations in activity of both substances should be considered and thoroughly evaluated before the clinical use of any surfactant-antibiotic mixtures [10].

The purpose of this study was to evaluate potential interactions between exogenous surfactant and three antibiotics (amikacin, cefepime, and colistimethate sodium) in vitro and, consequently, to determine a suitable combination(s) for subsequent investigations of surfactant/antibiotic experimental mixtures intended for local treatment of pneumonia. The study consisted of two parts: (1) assessment of influence of the antibiotics on surface-active properties of the exogenous surfactant and (2) assessment of influence of the exogenous surfactant on antibacterial properties of the antimicrobials.

\section{Materials and Methods}

2.1. Surfactant and Antibiotics. Sterile natural pulmonary surfactant preparation (Suzacrin, Docpharm, Ukraine) was used. This emulsion includes purified phospholipids (76\%; $50 \mathrm{mg}$ per $1 \mathrm{~mL}$ of emulsion) isolated from porcine lung homogenates and physiological solution as an excipient. Suzacrin contains surfactant-associated proteins B and C but is free of surfactant-proteins A and D.

The following antibiotics were utilized: amikacin (Amicil, Kievmedpreparat, Ukraine), cefepime (Cefepime, Nectar Lifesciences Ltd., India), and colistimethate sodium (Colomycin Injection, Forest Laboratories UK Ltd., UK). These antibacterial agents are currently recommended to treat pulmonary infections, caused by multidrug-resistant pathogens.

Solutions of antibiotics, emulsion of surfactant, and experimental mixtures of antibiotics in surfactant were freshly prepared in aseptic conditions using sterile $0.9 \% \mathrm{NaCl}$ solution immediately before testing.

Specific requirements were established to standardize experiments and to provide a balanced calculation of antibiotic and surfactant amount in experimental mixtures: quantitative ratio of antibiotic and surfactant should correspond to the ratio of mean daily doses recommended for adult male patient with a body mass of $70 \mathrm{~kg}$, that is, surfactant phospholipids $-700 \mathrm{mg}$, cefepime$2,000 \mathrm{mg}$, amikacin $-1,000 \mathrm{mg}$, and colistimethate sodium4,000,000 IU (320 mg).

2.2. Test Strains and Culture Media. The following test strains were provided by Crimean Sanitary-Epidemiological Station (Simferopol, Ukraine): Staphylococcus aureus ATCC25923, Klebsiella pneumoniae 3534/51, and Pseudomonas aeruginosa ATCC27853. Hottinger broth (Federal State Institution of Science "State Research Center of Applied Microbiology and Biotechnology," Obolensk, Russia; amine nitrogen concentration-132 mg\%) and meat-peptone agar (MPA;
State Pilot Factory of Bacterial Cultures of the Institute of Milk and Meat Technology, Kiev, Ukraine; amine nitrogen concentration- $2.7 \mathrm{mg} \%$ ) were used as culture media to investigate susceptibility of bacteria to antibiotics, surfactant, and surfactant-antimicrobial mixtures.

2.3. Assessment of Surface-Active Properties. The commercial surfactant preparation was diluted (reemulsified) in sterile $0.9 \% \mathrm{NaCl}$ solution (control) or similar volume of antibiotic solution (three test series) to produce equal final phospholipid concentration of $20.6 \mathrm{mg} / \mathrm{mL}$. The total volume of prepared experimental mixtures was $34 \mathrm{~mL}$ (a volume allowable for intratracheal administration to adult person within 24 hours). Final concentrations of antibiotics in surfactant-containing mixtures were as follows: amikacin$29.4 \mathrm{mg} / \mathrm{mL}$, colistimethate sodium-117,647 IU/mL, and cefepime- $58,8 \mathrm{mg} / \mathrm{mL}$.

Apart from the experimental series, five subsidiary series were investigated: undiluted surfactant preparation (phospholipid concentration-50 mg/mL), solutions of amikacin, colistimethate sodium, and cefepime in concentrations recommended for clinical use $(167 \mathrm{mg} / \mathrm{mL}, 500,000 \mathrm{IU} / \mathrm{mL}$ and $100 \mathrm{mg} / \mathrm{mL}$, resp.), and diluent ( $0.9 \% \mathrm{NaCl}$ solution).

The experiment was conducted at a temperature of $26^{\circ} \mathrm{C}$. Test suspensions, as well as control and subsidiary samples (five samples for every series), were foamed in sterile tubes with single-use medical syringes equipped with $23 \mathrm{G}$ injection needles. Foam was formed by triple aspiration and return of test material into a tube. The modified Pattle method $[14,15]$ was utilized to assess surface-active properties in stable foam. This method enables an evaluation of surface tension force dynamics on a liquid-gas interphase boundary according to the changes of air bubble diameter. For this purpose, hanging drops of obtained foam were examined in transmitted light using Olympus CX41 microscope equipped with Olympus C5050Z digital camera. One field of vision (200× magnification) uniformly filled with bubbles was photographed in every drop in automatic mode during $20 \mathrm{~min}$ with an interval of $30 \mathrm{sec}$. Thereby, each replication in a series included 41 shots (205 shots for each series composed of 5 replications). Obtained images (a total of 1,230 images) were analyzed using Olympus DP-Soft 3.11 application. Changes of 20 randomly chosen bubbles were monitored through all images of every replication. Bubble diameter $(\mu \mathrm{m})$ was measured for the first and final (obtained after $20 \mathrm{~min}$ ) images only. Totally, 1,200 bubble measurements were performed during this experiment. Stability index (SI) was calculated for each replication using the formula

$$
\mathrm{SI}=\frac{\sum d_{x}^{2}}{\sum d_{y}^{2}},
$$

when $d_{x}$ is a result of final measurement in a series (41st image) and $d_{y}$ is a result of first measurement (1st image).

2.4. Susceptibility Testing. Minimum inhibitory concentrations (MIC) of test substances were determined using conventional technique of serial dilution in Hottinger broth. 
TABLE 1: Foaming properties and stability index values for original preparations.

\begin{tabular}{|c|c|c|c|c|c|}
\hline $\begin{array}{l}\text { Foaming and surface activity } \\
\text { characteristics }\end{array}$ & $\begin{array}{l}\text { Surfactant } \\
\text { suspension }\end{array}$ & $\begin{array}{l}\text { Colistimethate } \\
\text { sodium solution }\end{array}$ & Amikacin solution & Cefepime solution & $\mathrm{NaCl}$ solution \\
\hline Foam properties & $\begin{array}{l}\text { Stable, } \\
>20 \mathrm{~min}\end{array}$ & $\begin{array}{c}\text { Stable, } \\
>20 \mathrm{~min}\end{array}$ & $\begin{array}{c}\text { Unstable, } \\
5-7 \mathrm{sec}\end{array}$ & $\begin{array}{l}\text { Unstable, } \\
10-15 \mathrm{sec}\end{array}$ & No foam \\
\hline \multicolumn{6}{|c|}{ Diameter of bubbles, $\mathrm{M} \pm \mathrm{m}(n), \mu \mathrm{m}:$} \\
\hline (a) start of experiment & $51.55 \pm 1.06(100)$ & $110.62 \pm 1.65(100)$ & - & - & - \\
\hline (b) end of experiment & $47.46 \pm 0.94(100)$ & $87.54 \pm 2.27(100)$ & - & - & - \\
\hline Difference between (a) and (b) & $4.09 \pm 0.29(100)$ & $23.07 \pm 1.58(100)$ & - & - & - \\
\hline Stability index (SI) & $0.85 \pm 0.02(5)$ & $0.65 \pm 0.03(5)$ & - & - & - \\
\hline
\end{tabular}

Note: $\mathrm{M} \pm \mathrm{m}$ : mean \pm standard error.

Each series of dilutions was investigated in two replications. The volume of liquid culture medium in each tube amounted to $1 \mathrm{~mL}$. During the antimicrobial activity assessment of antibiotics and mixtures of surfactant with antibiotics the quantity of surfactant and antibacterials in the initial tubes of series corresponded to aforecited mean daily dose ratios. When assessing surfactant's inherent influence on the bacterial cultures, surfactant concentration in the initial tube of series was 5,000 mg/L. Test substances were introduced into the tubes in a volume of $0.170 \mathrm{~mL}(0.100 \mathrm{~mL}$ of amikacin and colistimethate sodium solutions and $0.070 \mathrm{~mL}$ of surfactant suspension) or $0.135 \mathrm{~mL}(0.100 \mathrm{~mL}$ of cefepime solution and $0.035 \mathrm{~mL}$ of surfactant suspension). Then, these tubes were filled up with Hottinger broth till the final volume of $1 \mathrm{~mL}$. Maximum test concentration matched $1,000 \mathrm{mg} / \mathrm{L}$ for amikacin and cefepime, and $320 \mathrm{mg} / \mathrm{L}$ for colistimethate sodium. Maximum test concentration of surfactant in mixtures with antibiotics was $700 \mathrm{mg} / \mathrm{L}$ (for amikacin and colistimethate sodium) or $350 \mathrm{mg} / \mathrm{L}$ (for cefepime). Sequential twofold dilution of initial tube contents has been performed using the Hottinger broth. Then, the bacterial suspensions were introduced in all tubes in the volume of $0.1 \mathrm{~mL}$. The bacterial suspensions were prepared from diurnal bacterial cultures using the same broth. The density of introduced bacterial suspension amounted to $5 \times 10^{4} \mathrm{CFU} / \mathrm{mL}$. Obtained mixtures were incubated within 24 hours at $37^{\circ} \mathrm{C}$. Then, MIC was determined as a concentration of test substance in the last tube with clear broth in the presence of bacterial growth in control tube (free of test substances).

In order to determine a minimum bactericidal concentration (MBC; lowest concentration of substance that results in full inhibition of bacterial vital activity) $0.1 \mathrm{~mL}$ aliquots of test material from the tubes with transparent broth (with no visible bacterial growth) were transferred with a loop to MPAcontaining plates. Subsequent to incubation within 24 hours at $37^{\circ} \mathrm{C}, \mathrm{MBC}$ was determined as a minimum concentration of test substance that corresponded to the last plate in a series where microbial growth was completely absent.

2.5. Statistics. Most data are presented as a mean \pm standard error. Data were compared using the unpaired Student's $t$ test. A probability level of $P<0.05$ was considered to be statistically significant. Statistical analysis was performed using STATISTICA software version 10.0 (Stat Soft Inc., Tulsa, OK, USA).

\section{Results}

3.1. Assessment of Surface-Active Properties. The assessment of foaming properties of the officinal surfactant preparation, antibiotics (in therapeutic concentrations), and solvent demonstrated a wide range of surface activity characteristics (Table 1): from almost complete absence of foaming ability in normal saline to the formation of stable foam (which persisted $\geq 20 \mathrm{~min}$ ) in surfactant and colistimethate. Foaming properties of amikacin and cefepime solutions were rated as intermediate, but close to normal saline.

Bubbles of colistimethate sodium solution were significantly larger than bubbles of surfactant suspension both in the beginning $(P<0.001)$ and at the end of the experiment $(P<0.001)$. The diameter of colistimethate sodium and surfactant bubbles decreased during the experiment by $21 \%$ and $8 \%$, respectively $(P<0.001)$. SI values also suggested significantly higher surface activity of surfactant as compared with colistimethate sodium $(P<0.001)$.

Comparison of surfactant properties before and after dilution with normal saline (Tables 1 and 2) showed that 2.4-fold decrease of phospholipid concentration $(20.6 \mathrm{mg} / \mathrm{mL}$ versus initial $50 \mathrm{mg} / \mathrm{mL}$ ) does not result in significant changes of surface activity as per SI value $(P>0.05)$. However, the diameter of bubbles of diluted surfactant was significantly higher as compared with diameter of original surfactant suspension bubbles both in the beginning and at the end of the experiment $(P<0.001)$, thus suggesting a trend for reduction of surface activity.

As compared with control, experimental surfactant/amikacin mixture demonstrated smaller diameter of bubbles (Table 2) both in the beginning and at the end of the experiment $(P<0.001)$, as well as significantly lower tendency for reduction of bubble size in $20 \mathrm{~min}(P<0.001)$, whereas there were no substantial changes of SI $(P>0.05)$.

The surfactant/cefepime mixture also retained high surface activity as per SI values $(P>0.05)$. However, this mixture differed from the control by greater bubble diameter in the beginning of the experiment $(P<0.01)$ and somewhat more pronounced, or accelerated, reduction of bubble size $(P<$ $0.01)$. 
TABLE 2: Surface activity parameters of experimental mixtures.

\begin{tabular}{lcccc}
\hline Surface activity characteristics & $\begin{array}{c}\text { Surfactant }+\mathrm{NaCl} \\
(\text { control })\end{array}$ & $\begin{array}{c}\text { Surfactant + colistimethate } \\
\text { sodium }\end{array}$ & Surfactant + amikacin & Surfactant + cefepime \\
\hline \multicolumn{4}{c}{ Diameter of bubbles, $\mathrm{M} \pm \mathrm{m}(n), \mu \mathrm{m}:$} \\
(a) start of experiment & $77.98 \pm 2.03(100)$ & $83.23 \pm 3.23(100)$ & $56.04 \pm 2.01(100)$ & $86.55 \pm 2.17(100)$ \\
(b) end of experiment & $67.35 \pm 1.56(100)$ & $53.52 \pm 3.14(100)$ & $51.45 \pm 2.12(100)$ & $70.20 \pm 1.86(100)$ \\
Difference between (a) and (b) & $10.62 \pm 1.18(100)$ & $29.71 \pm 2.23(100)$ & $4.59 \pm 0.62(100)$ & $16.34 \pm 1.33(100)$ \\
Stability index (SI) & $0.76 \pm 0.04(5)$ & $0.49 \pm 0.06(5)$ & $0.85 \pm 0.05(5)$ & $0.67 \pm 0.04(5)$ \\
\hline
\end{tabular}

Note: $\mathrm{M} \pm \mathrm{m}$ : mean \pm standard error.

TABLE 3: Bacteriostatic and bactericidal characteristics of antibiotic solutions and experimental surfactant/antibacterial mixtures.

\begin{tabular}{|c|c|c|c|c|c|c|}
\hline \multirow{3}{*}{ Antibiotics and antibiotic/surfactant compositions } & \multicolumn{6}{|c|}{ MIC and MBC data $(\mathrm{mg} / \mathrm{L})$} \\
\hline & \multicolumn{2}{|c|}{ S. aureus } & \multicolumn{2}{|c|}{ K. pneumoniae } & \multicolumn{2}{|c|}{ P. aeruginosa } \\
\hline & MIC & MBC & MIC & MBC & MIC & $\mathrm{MBC}$ \\
\hline Amikacin & 3.9 & 3.9 & 1.9 & 1.9 & 3.9 & 3.9 \\
\hline Amikacin + surfactant & 3.9 & 3.9 & 1.9 & 1.9 & 3.9 & 3.9 \\
\hline Cefepime & $\leq 0.49$ & 1.9 & $>1,000$ & $>1,000$ & 3.9 & 500 \\
\hline Cefepime + surfactant & $\leq 0.49$ & 3.9 & $>1,000$ & $>1,000$ & $3.9(1.9)$ & 1,000 \\
\hline Colistimethate sodium & 80 & 80 & 20 & 160 & 20 & 160 \\
\hline Colistimethate sodium + surfactant & 20 & 80 & $20(10)$ & 160 & 10 & 160 \\
\hline
\end{tabular}

Notes: MBC: minimum bactericidal concentration; MIC: minimum inhibitory concentration. Additional MIC values are presented in round parentheses when different results were obtained for distinct replications.

There was no statistically significant difference in initial bubble size between surfactant/colistimethate sodium and control mixtures $(P>0.05)$, whereas experimental mixture bubbles had substantially smaller diameter than surfactant bubbles at the end of the experiment $(P<0.001)$. Therefore, a trend for bubble diameter decrease was more pronounced after antibiotic addition $(P<0.001)$. Relevant SI decrease was observed in this experiment as compared with control $(P<0.01)$, original surfactant preparation $(P<0.001)$, or colistimethate sodium solution $(P<0.05)$ (Table 2$)$.

3.2. Susceptibility Testing. The exogenous surfactant preparation was devoid of apparent intrinsic antimicrobial effects as for S. aureus, K. pneumoniae, and P. aeruginosa cultures.

Amikacin demonstrated its efficiency against all three bacterial strains. This antibiotic completely inhibited growth of $S$. aureus and P. aeruginosa in 256-fold dilution, as well as growth of K. pneumoniae-in 526-fold dilution, that is, in concentration of 3.9 and $1.9 \mathrm{mg} / \mathrm{L}$, respectively (Table 3). Antibacterial properties of amikacin were not affected when combined with surfactant suspension-MIC and MBC remained on the same level.

Cefepime exhibited most pronounced antistaphylococcal activity $(\mathrm{MIC} \leq 0.49 \mathrm{mg} / \mathrm{L}, \mathrm{MBC}=1.9 \mathrm{mg} / \mathrm{L})$. However, when tested against $P$. aeruginosa, $\mathrm{MBC}$ of cefepime was significantly (128-fold) higher than MBC of amikacin, whereas antipseudomonal MICs of these antibiotics were equally substantial. K. pneumoniae demonstrated full resistance to cefepime (bacterial growth was observed in all dilutions both in broth and after reinoculation on agar). Suspending of exogenous surfactant in cefepime resulted in slight decrease of bactericidal activity against $S$. aureus and $P$. aeruginosa cultures.

Colistimethate sodium had significantly lower antibacterial activity as compared to amikacin (against all three cultures) and cefepime (against $S$. aureus). The ability of colistimethate sodium to inhibit growth of $K$. pneumoniae and $P$. aeruginosa was moderate but higher than in cefepime. When combined with surfactant, bacteriostatic effect of colistimethate increased 4 - and 2-fold for $S$. aureus and $P$. aeruginosa, respectively, though remaining on relatively low level (20 mg/L and $10 \mathrm{mg} / \mathrm{L})$, while bactericidal activity was unchanged.

\section{Discussion and Conclusions}

It has been previously shown that interactions between antibiotics and surfactant may exist, and these interactions may influence efficiency of either substances [7, 10]. The purpose of this study was to assess potential mutual influence of natural surfactant preparation and three antibiotics (amikacin, cefepime, and colistimethate sodium) in vitro and to identify appropriate combination(s) for subsequent investigations of topical surfactant/antibiotic therapy in experimental acute bacterial pulmonary infection. Potential limitations of this study include restrictions as regards extrapolation of the results to other exogenous surfactant preparations due to differences in chemical composition of currently available surfactant products.

In the first part of the study it has been revealed that addition of the amikacin or cefepime to the surfactant had no significant influence on the surface-active properties 
of the latter, whereas obvious reduction of this feature was detected when mixing surfactant with colistimethate sodium. Considering the ability of colistimethate sodium to disrupt phospholipid bilayer of bacterial cell envelope [16], supposedly the observed effect may be caused by specific interaction of the colistimethate sodium with phospholipids of the surfactant.

The results of microbiological part of the study suggest that the surfactant preparation was devoid of intrinsic antibacterial activity. When mixed with antibiotics, the surfactant either had no impact on antimicrobial activity of the latter (e.g., amikacin entirely preserved its properties) or exerted mild to moderate influence. Specifically, there was a trend for decrease of cefepime bactericidal activity and increase of colistimethate sodium bacteriostatic activity against $S$. aureus and $P$. aeruginosa.

Interaction between exogenous surfactant and antibiotics was previously studied by van't Veen et al. [7, 10]. They have determined that surface-active properties of bovine pulmonary surfactant were not altered after addition of ceftazidime or pentamidine, whereas activity of surfactant was depressed with amphotericin B and amoxicillin. Surfactant surface-active properties in tobramycin/surfactant mixture were dependent on the type of solvent used. Surface-active properties of the surfactant were depressed when saline was utilized as a solvent but unaffected when tobramycin was mixed with surfactant in $0.2 \mathrm{M} \mathrm{NaHCO}_{3}$ buffer [10].

An ability of exogenous surfactant to suppress activity of certain antibiotics was demonstrated in vitro [7] in terms of tobramycin, whereas bactericidal activity of amoxicillin and ceftazidime was not altered. The authors have explained the depression of tobramycin antibacterial activity against $K$. pneumoniae, $P$. aeruginosa, S. aureus, and Streptococcus pneumoniae by the ability of aminoglycosides to bind negatively charged phospholipids contained in surfactant preparation and assumed that an excess of phospholipids can inactivate almost all the aminoglycosides [5, 7].

In contrast to the aforecited tobramycin/surfactant compatibility issues $[7,10]$, the results of present study indicate that activity of amikacin (another aminoglycoside antibiotic) was not suppressed by the surfactant preparation, and surface-active properties of the surfactant appeared unaffected when saline was used as a solvent for antibiotic, suggesting that not all aminoglycosides possess negative interaction with exogenous surfactant, at least considering specific features of given experiments (different surfactant preparation, different concentration of surfactant in the mixture, etc.).

Assuming relatively low activity of the colistimethate sodium against tested bacterial cultures, as well as noticeable reduction of surfactant surface activity in the surfac$\operatorname{tant} /$ colistimethate sodium mixture, the use of this composition in subsequent studies in animals infected with $S$. aureus, $K$. pneumoniae, and $P$. aeruginosa appears to be unreasonable. Conversely, it is advisable to use the surfactant/amikacin composition for experimental studies in animals infected with given strains of $S$. aureus, $K$. pneumoniae, and $P$. aeruginosa, and the surfactant/cefepime composition for experimental staphylococcal infection in laboratory animals.
In summary, the study showed that an addition of therapeutic concentrations of the amikacin and cefepime to the exogenous surfactant preparation does not cause a decrease of surfactant surface activity in vitro, whereas significant suppression of both surfactant and colistimethate sodium surface-active properties is evident when mixed in one volume. Addition of the surfactant suspension to amikacin had no influence on high antimicrobial activity of the latter, whereas suspending surfactant with cefepime or colistimethate sodium resulted in a trend for decrease of cefepime bactericidal activity and increase of colistimethate sodium bacteriostatic activity against $S$. aureus and $P$. aeruginosa. Obtained data confirm the necessity of surfactantantibacterial interaction assessment before utilization of any surfactant/antibiotic mixtures in clinical settings and in preclinical animal studies and show that further investigation of combined surfactant/antibacterial therapy should include an evaluation of the surfactant/amikacin composition in animals with bacterial pneumonia.

\section{Conflict of Interests}

The author, Alexei Birkun, declares that there is no conflict of interests regarding the publication of this paper.

\section{References}

[1] D. R. Baldwin, D. Honeybourne, and R. Wise, "Pulmonary disposition of antimicrobial agents: in vivo observations and clinical relevance," Antimicrobial Agents and Chemotherapy, vol. 36, no. 6, pp. 1176-1180, 1992.

[2] D. J. Touw, R. W. Brimicombe, M. E. Hodson, H. G. M. Heijerman, and W. Bakker, "Inhalation of antibiotics in cystic fibrosis," European Respiratory Journal, vol. 8, no. 9, pp. 15941604, 1995.

[3] J. D. Brain, D. E. Knudson, S. P. Sorokin, and M. A. Davis, "Pulmonary distribution of particles given by intratracheal instillation or by aerosol inhalation," Environmental Research, vol. 11, no. 1, pp. 13-33, 1976.

[4] J. S. Ilowite, J. D. Gorvoy, and G. C. Smaldone, "Quantitative deposition of aerosolized gentamicin in cystic fibrosis," American Review of Respiratory Disease, vol. 136, no. 6, pp. 1445-1449, 1987.

[5] J. J. Haitsma, U. Lachmann, and B. Lachmann, "Exogenous surfactant as a drug delivery agent," Advanced Drug Delivery Reviews, vol. 47, no. 2-3, pp. 197-207, 2001.

[6] V. S. Kharasch, T. D. Sweeney, J. Fredberg et al., "Pulmonary surfactant as a vehicle for intratracheal delivery of technetium sulfur colloid and pentamidine in hamster lungs," American Review of Respiratory Disease, vol. 144, no. 4, pp. 909-913, 1991.

[7] A. van't Veen, J. W. Mouton, D. Gommers, J. A. J. W. Kluytmans, P. Dekkers, and B. Lachmann, "Influence of pulmonary surfactant on in vitro bactericidal activities of amoxicillin, ceftazidime, and tobramycin," Antimicrobial Agents and Chemotherapy, vol. 39, no. 2, pp. 329-333, 1995.

[8] L. A. J. M. Creuwels, L. M. G. van Golde, and H. P. Haagsman, "The pulmonary surfactant system: biochemical and clinical aspects," Lung, vol. 175, no. 1, pp. 1-39, 1997.

[9] L. M. G. van Golde, J. J. Batenburg, and B. Robertson, “The pulmonary surfactant system: biochemical aspects and functional 
significance," Physiological Reviews, vol. 68, no. 2, pp. 374-453, 1988.

[10] A. van't Veen, D. Gommers, J. W. Mouton, J. A. J. W. Kluytmans, E. J. Krijt, and B. Lachmann, "Exogenous pulmonary surfactant as a drug delivering agent: influence of antibiotics on surfactant activity," British Journal of Pharmacology, vol. 118, no. 3, pp. 593598, 1996.

[11] A. van't Veen, J. W. Mouton, D. Gommers, and B. Lachmann, "Pulmonary surfactant as vehicle for intratracheally instilled tobramycin in mice infected with Klebsiella pneumoniae," British Journal of Pharmacology, vol. 119, no. 6, pp. 1145-1148, 1996.

[12] A. van't Veen, D. Gommers, S. J. C. Verbrugge et al., "Lung clearance of intratracheally instilled $99 \mathrm{~m}$ Tc-tobramycin using pulmonary surfactant as vehicle," British Journal of Pharmacology, vol. 126, no. 5, pp. 1091-1096, 1999.

[13] G. Stichtenoth, B. Linderholm, M. H. Björkman, G. Walter, T. Curstedt, and E. Herting, "Prophylactic intratracheal polymyxin $\mathrm{B} /$ surfactant prevents bacterial growth in neonatal Escherichia coli pneumonia of rabbits," Pediatric Research, vol. 67, no. 4, pp. 369-374, 2010.

[14] R. E. Pattle, C. C. Kratzing, C. E. Parkinson et al., "Maturity of fetal lungs tested by production of stable microbubbles in amniotic fluid," British Journal of Obstetrics and Gynaecology, vol. 86, no. 8, pp. 615-622, 1979.

[15] M. A. Zavalij, A. G. Balabantsev, A. K. Zagorul'ko, A. A. Zavalij, A. N. Orel, and S. V. Shapran, "Method for evaluation of efficiency of bronchoalveolar protective complex use in treatment of acute suppurative sinuitis in experiment," Journal of Ear, Nose and Throat Diseases, vol. 5, pp. 68-69, 2008 (Russian).

[16] C. Mestres, M. A. Alsina, M. A. Busquets, I. Murányi, and F. Reig, "Interaction of colistin with lipids in liposomes and monolayers," International Journal of Pharmaceutics, vol. 160, no. 1, pp. 99-107, 1998. 

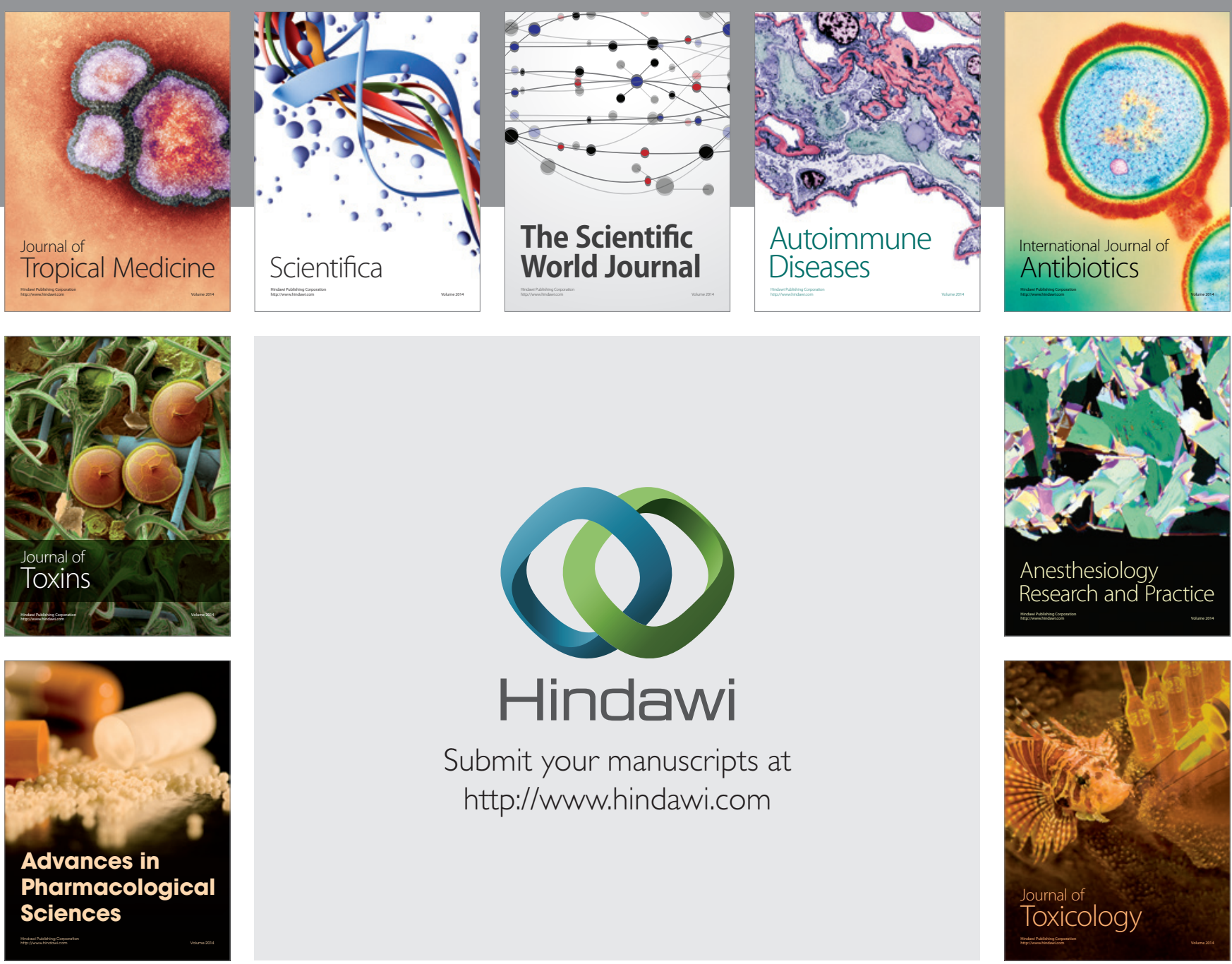

\section{Hindawi}

Submit your manuscripts at

http://www.hindawi.com
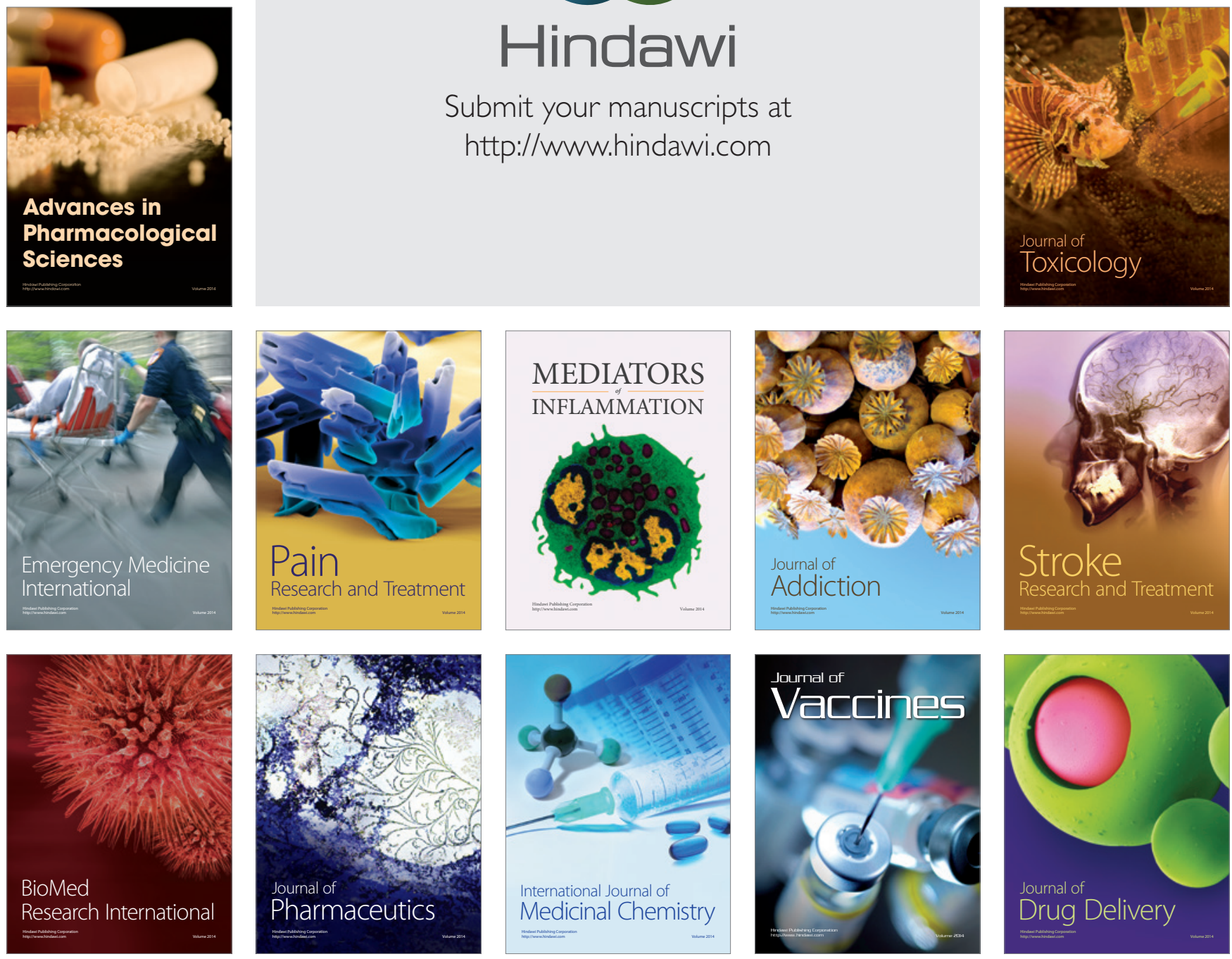